\title{
Análise Comparativa dos Custos de Implantação de Redes Simples e Duplas para Distribuição de Água Empregando Tubos de PVC
}

\author{
Comparative Analysis of Deployment Costs of Simple and Double Network for Water \\ Distribution Using PVC pipes
}

\begin{abstract}
Análisis comparativo de los costos de despliegue de red simples y dobles para la
\end{abstract} distribución de agua mediante tuberías de PVC

André de Moura Leite Aluno de graduação do Curso de Engenharia Civil, UFSCar, Brasil talciclano@usp.br

Erich Kellner Professor Associado, UFSCar, Brasil. erich.kellner@ufscar.br)

Jorge Akutsu Professor Doutor, UFSCar, Brasil. akutsu@ufscar.br 


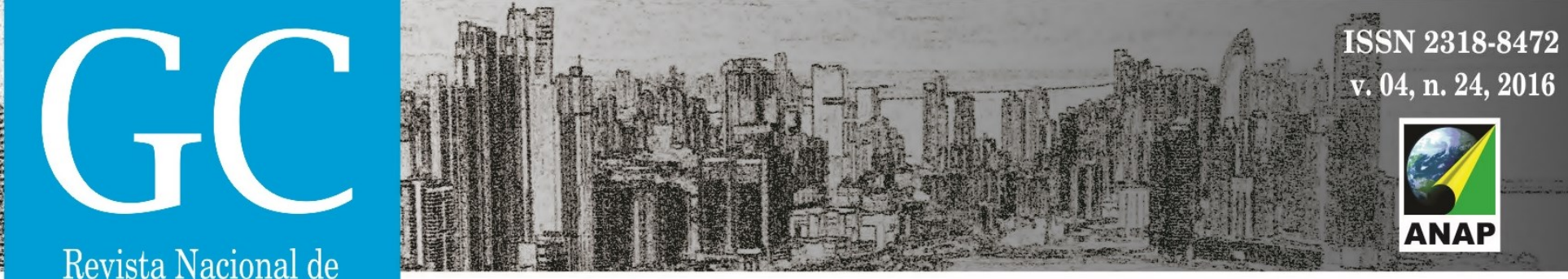

Gerenciamento de Cidades

\section{INTRODUÇÃO}

A rede de distribuição de água é composta por um conjunto de tubulações e conexões que deve garantir a condução da água potável em quantidade e pressões adequadas sem alterar sua qualidade.

Assim como qualquer obra, o projeto e a construção de uma rede de distribuição de água requer planejamento devendo ser observados seus aspectos construtivos e atendimento as especificidades de cada projeto.

De acordo com a NBR 12.218/1994 - ABNT(1994), que trata do projeto de redes de distribuição de água para abastecimento público, a execução de redes duplas de abastecimento de água é prevista em dois casos: em ruas principais de tráfego intenso e quando a análise do projeto demonstrar que a rede dupla seja mais econômica.

Neste trabalho, para a análise de custos, foram considerados os aspectos construtivos, serviços e insumos necessários para a execução de redes simples e duplas, assim como as ligações prediais, empregando-se as planilhas do SINAPI - Sistema Nacional de Pesquisa de Custos e Índices da Construção Civil, para o Estado de São Paulo.

Este trabalho tem o intuito de contribuir para a tomada de decisão, sob a ótica econômica, da viabilidade ou não da implantação de redes duplas de distribuição de água, lançando luz à NBR $12.218 / 1994$.

\section{OBJETIVOS}

O objetivo geral deste artigo foi o de analisar os custos de implantação de redes públicas de distribuição de água simples e duplas, empregando tubos de PVC PBA, de maneira a subsidiar, técnica e economicamente, baseado na orientação estabelecida na NBR 12.218/1994.

Visando atingir o objetivo principal, foram estabelecidos os seguintes objetivos específicos:

- Quantificação dos insumos e serviços envolvidos nos processos construtivos das redes de distribuição de água;

- Valoração dos quantitativos identificados segundo preços estabelecidos no Sistema Nacional de Preços - SINAPI

- Analise das condições de menor custo para implantação da rede pública de distribuição de água.

\section{MATERIAL E MÉTODOS}

Nesta seção foram apresentados e discutidos os métodos empregados para o desenvolvimento do trabalho proposto, tendo sido optado por apresentá-los como se seguem.

\subsection{COMPOSIÇÃO DOS CUSTOS DE IMPLANTAÇÃO}

Os custos envolvidos na implantação de uma rede de distribuição de água são compostos pelos custos dos materiais envolvidos, bem como pelos serviços realizados para sua construção.

A composição dos custos de implantação e em especial os custos de serviços, dependem das características do terreno onde a rede será implantada, bem como das características da seção 


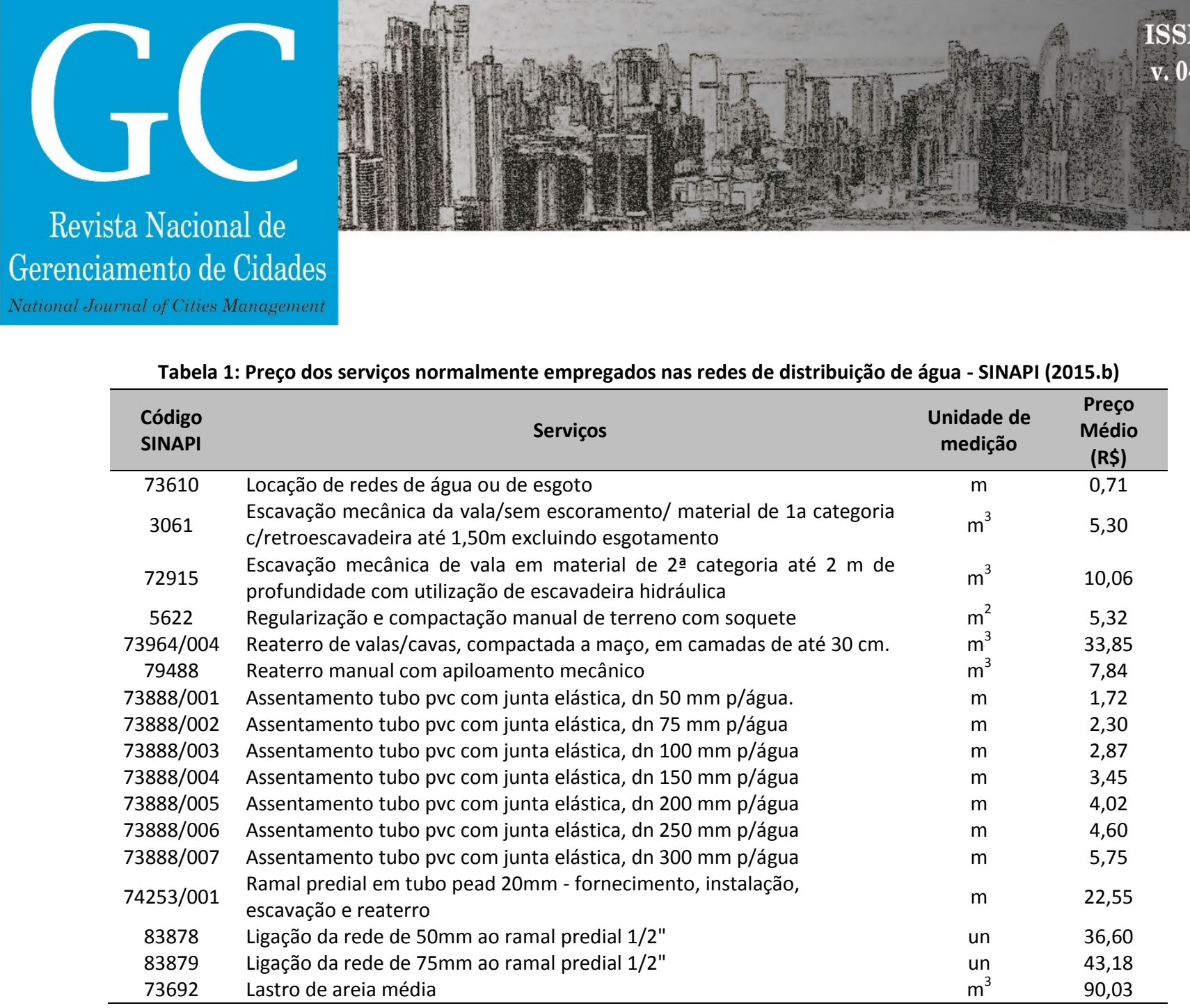

Fonte: adaptado de SINAPI (2015.b)

\subsubsection{Custos de Materiais}

Com relação aos materiais empregados na construção da rede de distribuição de água, foram considerados os seguintes insumos:

- Pasta lubrificante para aplicação nas juntas elásticas;

- Anel de borracha;

- Tubulações em PVC PBA - Classe 15;

- Colar de tomada para interligação da rede pública às ligações prediais;

- Tubo PEAD para ligações prediais.

A partir dos insumos acima listados, procurou-se junto ao SINAPI (2015.a) as unidades de medição e os custos médios considerados, conforme apresentados na Tabela 2. 


\section{Revista Nacional de}

Gerenciamento de Cidades

Tabela 3: Consumo de pasta lubrificante para junta elástica em função do diâmetro nominal (DN)

\begin{tabular}{ccc}
\hline & \multicolumn{3}{c}{$\begin{array}{c}\text { Consumo de Pasta Lubrificante } \\
\text { (g/junta) }\end{array}$} \\
\cline { 2 - 3 } & PVC PBA & PVC DEFoFo \\
\hline 50 & 13,5 & - \\
15 & 22,5 & 31,5 \\
100 & 27,0 & 58,5 \\
200 & - & 76,5 \\
250 & - & 112,5 \\
300 & - & 153,0 \\
\hline
\end{tabular}

Fonte: FORTILIT (s.d.)

A profundidade da vala, considerando solo de primeira categoria, pode ser expressa pela Equação (03):

$$
H_{v}=\left(h_{\min }+\emptyset_{e}\right)
$$

sendo $H_{v}$ a profundidade da vala para solo de primeira categoria $(\mathrm{m}) ; L_{v}$ a largura da vala $(\mathrm{m})$ admitida igual a $0,60 \mathrm{~m} ; h_{\min }$ a altura mínima para recobrimento da tubulação $(\mathrm{m}) ; \emptyset_{e} \mathrm{o}$ diâmetro externo da tubulação $(m)$ conforme expresso na Tabela .

Tabela 4: Dimensões características das tubulações de PVC PBA

\begin{tabular}{|c|c|c|c|c|c|c|c|}
\hline \multirow{2}{*}{$\begin{array}{c}\text { Classe de } \\
\text { Pressão }\end{array}$} & \multicolumn{6}{|c|}{ Dimensões Características (mm) } & \multirow{2}{*}{$\begin{array}{l}\text { Massa } \\
(\mathrm{kg} / \mathrm{m})\end{array}$} \\
\hline & DN & De & Di & L & B & e & \\
\hline 12 & 50 & (60) 60,4 & 55,0 & & 85 & 2,7 & 0,73 \\
\hline \multirow[t]{2}{*}{$(0,6 \mathrm{MPa})$} & 75 & (85) 85,6 & 77,8 & 6000 & 100 & 3,9 & 1,50 \\
\hline & 100 & (110) 110,6 & 100,6 & & 120 & 5,0 & 2,44 \\
\hline 15 & 50 & (60) 60,4 & 53,8 & & 85 & 3,3 & 0,87 \\
\hline \multirow[t]{2}{*}{$(0,75 \mathrm{MPa})$} & 75 & (85) 85,6 & 76,2 & 6000 & 100 & 4,7 & 1,76 \\
\hline & 100 & (110) 110,6 & 98,4 & & 120 & 6,1 & 2,92 \\
\hline \multirow{3}{*}{$\begin{array}{c}20 \\
(1,0 \mathrm{MPa})\end{array}$} & 50 & (60) 60,4 & 51,8 & & 85 & 4,3 & 1,10 \\
\hline & 75 & (85) 85,6 & 73,4 & 6000 & 100 & 6,1 & 2,21 \\
\hline & 100 & (110) 110,6 & 95,0 & & 120 & 7,8 & 3,67 \\
\hline
\end{tabular}

Fonte: adaptado de AMANCO (2014).

Nota: DN - Diâmetro Nominal $(\mathrm{mm})$; De - Diâmetro Externo $(\mathrm{mm})$; Di - Diâmetro Interno $(\mathrm{mm})$; L - Comprimento $(\mathrm{mm}) ; \mathrm{B}$ - Comprimento da Bolsa (mm); e - Espessura da parede (mm)

O volume escavado da vala de primeira categoria $(\mathrm{m})$ pode ser estimado pela Equação (04):

$$
V_{e}=L_{v} \cdot H_{v} \cdot Z
$$

sendo $V_{e}$ o volume escavado da vala $\left(\mathrm{m}^{3}\right) ; L_{v}$ a largura da vala $(\mathrm{m}) ; H_{v}$ a profundidade da vala $(\mathrm{m}) ; Z$ o comprimento do trecho $(\mathrm{m})$.

A área para regularização e compactação do fundo da vala pode ser estimada pela Equação (05):

$$
A R F V=L_{v} \cdot Z
$$

sendo $A R F V$ a área para regularização do fundo da vala $\left(\mathrm{m}^{2}\right), L_{v}$ a largura da vala $(\mathrm{m}) ; Z$ o comprimento do trecho $(\mathrm{m})$.

$O$ volume de aterro com compactação manual realizado até $0,30 \mathrm{~m}$ acima da geratriz superior da tubulação pode ser estimado pela Equação (06): 


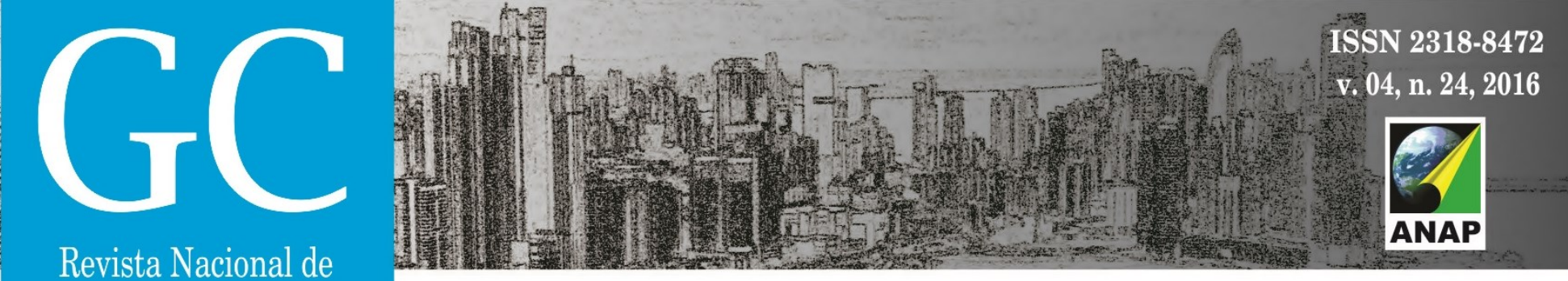

Gerenciamento de Cidades

$$
V A C M=(1+\varepsilon) \cdot\left[L_{v} \cdot\left(0,30+\emptyset_{e}\right)-\left(\frac{\pi \cdot \emptyset_{e}^{2}}{4}\right)\right] \cdot Z
$$

sendo VACM o volume de aterro com compactação manual $\left(\mathrm{m}^{3}\right)$; $\varepsilon$ o grau de empolamento (dec.), conforme Tabela $5 ; L_{v}$ a largura da vala $(\mathrm{m}) ; \emptyset_{\mathrm{e}}$ o diâmetro externo da tubulação $(\mathrm{m})$, conforme Tabela ; $Z$ o comprimento do trecho considerado (m).

\begin{tabular}{cc} 
Tabela 5: Empolamento em função do tipo de material escavado \\
\hline Material Escavado & Empolamento \\
\hline Solo argiloso & $40 \%$ \\
Terra Comum & $25 \%$ \\
Solo arenoso seco & $12 \%$ \\
\hline
\end{tabular}

Fonte: adaptado de São Paulo (2006).

O volume de aterro com compactação mecânica pode ser estimado pela Equação (08):

$$
\operatorname{VACMec}=(1+\varepsilon) \cdot\left[H_{v}-\left(0,30+\emptyset_{e}\right)\right] \cdot L_{v} \cdot Z
$$

sendo VACMec o volume de aterro com compactação mecanizada $\left(\mathrm{m}^{3}\right) ; \varepsilon$ o grau de empolamento (dec.), conforma Tabela $5 ; L_{v}$ a largura da vala $(\mathrm{m}) ; \emptyset_{\mathrm{e}}$ o diâmetro externo da tubulação $(\mathrm{m})$, conforme Tabela ; $Z$ o comprimento do trecho considerado $(\mathrm{m})$.

\section{RESULTADOS E DISCUSSÃO}

Nesta seção são apresentados e discutidos os resultados obtidos das análises realizadas no desenvolvimento do projeto proposto.

\subsection{COMPOSIÇÃo dOS CUSTOS PARA IMPLANTAÇÃO DAS REDES DE DISTRIBUIÇÃO}

Nesta seção foram apresentados os custos para implantação das redes de distribuição de água potável implantadas como redes simples, redes duplas, assim como os custos das ligações em cada um dos tipos de redes de distribuição.

\subsubsection{Custos para Implantação de redes duplas}

As redes de distribuição duplas são aquelas assentadas nos dois passeios existentes na via, conforme desenho esquemático representado pela Tabela 6 contém os quantitativos de serviços e materiais, em função dos diâmetros nominais das redes de distribuição de água assentadas nos dois passeios. 


\section{Revista Nacional de}

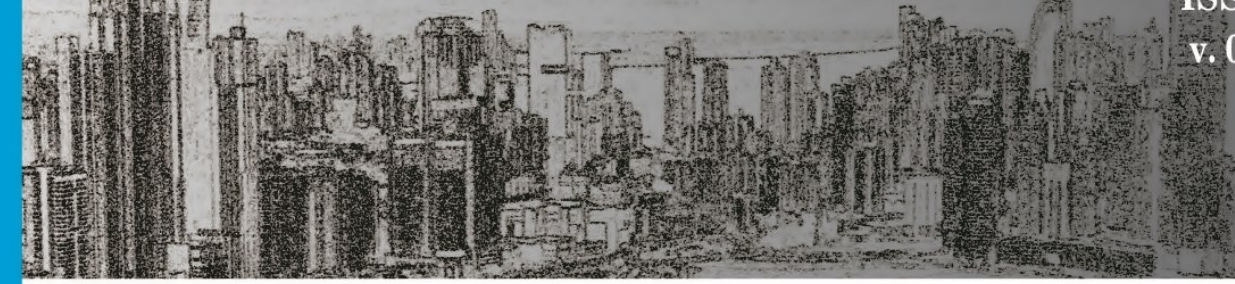

Tabela 6: Quantitativos esperados para construção de trecho de $6 \mathrm{~m}$ de rede de distribuição de água assentada nos dois passeios e em função do diâmetro nominal da rede

\begin{tabular}{|c|c|c|c|c|}
\hline \multirow{2}{*}{\multicolumn{2}{|c|}{ Quantitativos esperados para cada $6 \mathrm{~m}$ de rede dupla }} & \multicolumn{3}{|c|}{ DN } \\
\hline & & 50 & 75 & 100 \\
\hline \multicolumn{2}{|c|}{ Diâmetro Externo - De (mm) } & 60,4 & 85,6 & 110,6 \\
\hline \multirow{3}{*}{ 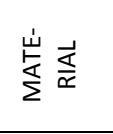 } & Tubulação PVC (m) & 12,0 & 12,0 & 12,0 \\
\hline & Anéis de borracha (un.) & 2 & 2 & 2 \\
\hline & Pasta Lubrificante (g) & 27,0 & 45,0 & 54,0 \\
\hline \multirow{4}{*}{ 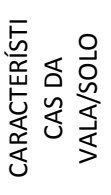 } & Profundidade da Vala - Hv (m) & 0,76 & 0,79 & 0,81 \\
\hline & Largura da vala - Lv (m) & 0,60 & 0,60 & 0,60 \\
\hline & Tipo de solo: & \multicolumn{3}{|c|}{ 1a Categoria } \\
\hline & Empolamento $-\varepsilon(\%)$ & 25 & 25 & 25 \\
\hline \multirow{6}{*}{ 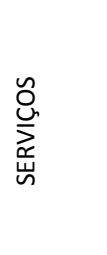 } & Locação da rede de água $(\mathrm{m})$ & 12,0 & 12,0 & 12,0 \\
\hline & Volume Escavado - Ve $\left(\mathrm{m}^{3}\right)$ & 7,64 & 7,82 & 8,00 \\
\hline & Regularização e compactação do fundo de vala $\left(\mathrm{m}^{2}\right)$ & 7,2 & 7,2 & 7,2 \\
\hline & Assentamento da Tubulação (m) & 12 & 12 & 12 \\
\hline & Volume de aterro com apiloamento manual - VACM $\left(\mathrm{m}^{3}\right)$ & 3,20 & 3,38 & 3,56 \\
\hline & Volume de aterro com compactação mecânica-VACMec $\left(\mathrm{m}^{3}\right)$ & 6,30 & 6,30 & 6,30 \\
\hline
\end{tabular}

A partir da determinação dos quantitativos envolvidos para a implantação de rede de distribuição de água assentada nos dois passeios da via, aplicou-se os custos estabelecidos pelo SINAPI (2015.a) e SINAPI (2015.b), conforme apresentados na Tabela 7, de maneira a determinar os custos de serviços e materiais, respectivamente.

A Tabela apresenta os custos determinados para implantação de redes de distribuição de água, assentada nos dois passeios e em função do diâmetro nominal da rede.

Tabela 7: Composição dos custos envolvidos no assentamento de redes de distribuição de água nos dois passeios em função do Diâmetro Nominal (DN) da rede

\begin{tabular}{|c|c|c|c|c|}
\hline & \multirow{2}{*}{ Custos envolvidos } & \multicolumn{3}{|c|}{ DN } \\
\hline & & 50 & 75 & 100 \\
\hline \multirow{5}{*}{ 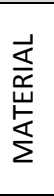 } & Tubulação PVC (m) & 111,24 & 209,16 & 375,72 \\
\hline & Anéis de borracha (un.) & 2,72 & 8,96 & 7,00 \\
\hline & Pasta Lubrificante (g) & 0,64 & 1,04 & 1,26 \\
\hline & Sub-Total (R\$ por $6 \mathrm{~m})$ & 114,60 & 219,16 & 383,98 \\
\hline & Relação entre o custo do material e o custo total (\%) & $32,9 \%$ & $47,0 \%$ & $59,5 \%$ \\
\hline \multirow{10}{*}{ 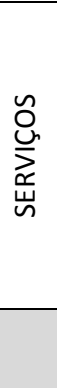 } & Locação da rede de distribuição de água $(\mathrm{m})$ & 8,52 & 8,52 & 8,52 \\
\hline & Volume Escavado - Ve $\left(\mathrm{m}^{3}\right)$ & 29,04 & 30,10 & 30,96 \\
\hline & Regularização e compactação do fundo de vala $\left(\mathrm{m}^{2}\right)$ & 38,30 & 38,30 & 38,30 \\
\hline & Assentamento da Tubulação $(\mathrm{m})$ & 20,64 & 27,60 & 34,44 \\
\hline & Volume de aterro com apiloamento manual - VACM $\left(\mathrm{m}^{3}\right)$ & 108,32 & 114,42 & 120,50 \\
\hline & Volume de aterro com compactação mecânica-VACMec $\left(\mathrm{m}^{3}\right)$ & 28,22 & 28,22 & 28,22 \\
\hline & Sub-Total (R\$ por $6 \mathrm{~m})$ & 233,04 & 247,16 & 260,94 \\
\hline & Relação entre o custo dos serviços e o custo total (\%) & $67,1 \%$ & $53,0 \%$ & $40,5 \%$ \\
\hline & \multirow{2}{*}{\begin{tabular}{ll} 
Custo Total & Por $6 \mathrm{~m}(\mathrm{R} \$)$ \\
\cline { 2 - 2 } & Linear $(\mathrm{R} \$ \mathrm{~m})$
\end{tabular}} & 347,64 & 466,32 & 644,92 \\
\hline & & 57,94 & 77,72 & 107,49 \\
\hline
\end{tabular}




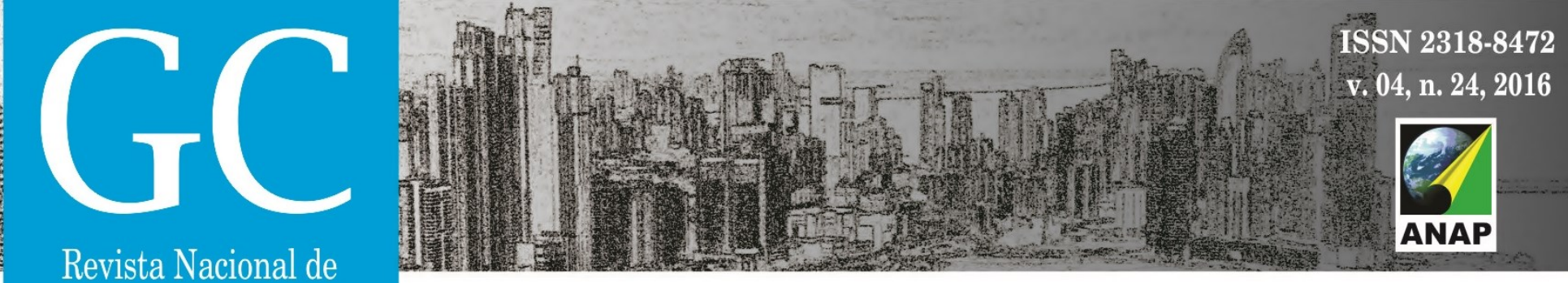

Gerenciamento de Cidades

\subsubsection{Custos para implantação de redes simples}

As redes de distribuição de água simples são aquelas assentadas em um dos terços do leito carroçável e responsáveis pelo atendimento das ligações dispostas em ambos os lados via, conforme desenho esquemático representado pela Tabela 8.

A Tabela contém os quantitativos de serviços e materiais, em função dos diâmetros nominais das redes de distribuição de água assentadas no terço do leito carroçável.

Tabela 8: Quantitativos esperados para construção de trecho de $6 \mathrm{~m}$ de rede de distribuição de água assentada no terço do leito carroçável e em função do diâmetro nominal da rede

\begin{tabular}{|c|c|c|c|c|}
\hline \multirow{2}{*}{\multicolumn{2}{|c|}{ Quantitativos esperados a cada barra de $6 \mathrm{~m}$ assentada no terço do leito carroçável }} & \multicolumn{3}{|c|}{ DN } \\
\hline & & 50 & 75 & 100 \\
\hline \multicolumn{2}{|c|}{ Diâmetro Externo - De (mm) } & 60,4 & 85,6 & 110,6 \\
\hline \multirow{3}{*}{ 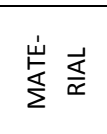 } & Tubulação PVC (m) & 6,0 & 6,0 & 6,0 \\
\hline & Anéis de borracha (un.) & 1 & 1 & 1 \\
\hline & Pasta Lubrificante (g) & 13,5 & 22,5 & 27,0 \\
\hline \multirow{4}{*}{ 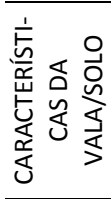 } & Profundidade da Vala $-\mathrm{Hv}(\mathrm{m})$ & 1,06 & 1,09 & 1,11 \\
\hline & Largura da vala - Lv (m) & 0,60 & 0,60 & 0,60 \\
\hline & Tipo de solo: & \multicolumn{3}{|c|}{ 1a Categoria } \\
\hline & Empolamento - $\varepsilon(\%)$ & 25 & 25 & 25 \\
\hline \multirow{6}{*}{ 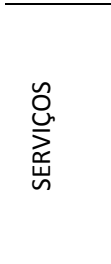 } & Locação da rede de água $(\mathrm{m})$ & 6,0 & 6,0 & 6,0 \\
\hline & Volume Escavado - Ve $\left(\mathrm{m}^{3}\right)$ & 3,82 & 3,91 & 4,00 \\
\hline & Regularização e compactação do fundo de vala $\left(\mathrm{m}^{2}\right)$ & 3,6 & 3,6 & 3,6 \\
\hline & Assentamento da Tubulação $(\mathrm{m})$ & 6,0 & 6,0 & 6,0 \\
\hline & Volume de aterro com apiloamento manual - VACM $\left(\mathrm{m}^{3}\right)$ & 1,60 & 1,69 & 1,78 \\
\hline & Volume de aterro com compactação mecânica-VACMec $\left(\mathrm{m}^{3}\right)$ & 3,15 & 3,15 & 3,15 \\
\hline
\end{tabular}

A partir da determinação dos quantitativos envolvidos para a implantação de rede de distribuição de água assentada no terço do leito carroçável, aplicou-se os custos estabelecidos pelo SINAPI (2015.a) e SINAPI (2015.b), conforme apresentados na Tabela 8 de maneira a determinar os custos de serviços e materiais, respectivamente.

Na Tabela 9 apresentam-se os custos determinados para implantação de redes de distribuição de água, assentada no terço do leito carroçável, em função do diâmetro nominal da rede. 


\section{Revista Nacional de}

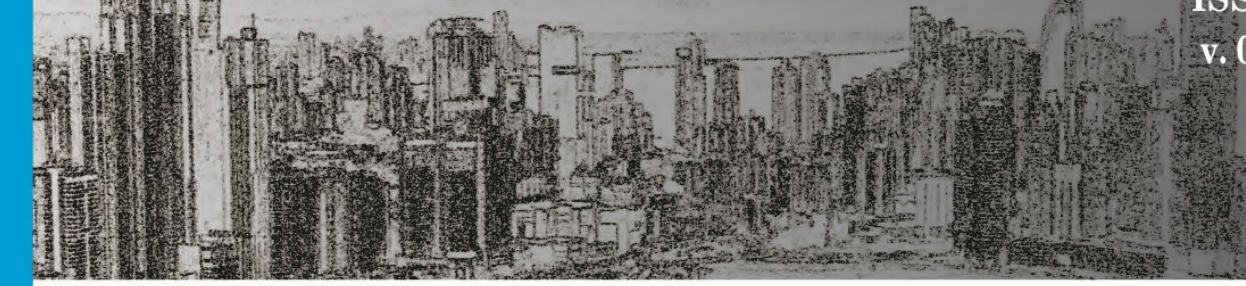

Tabela 9: Composição dos custos envolvidos no assentamento de redes de distribuição de água assentada no terço do leito carroçável, em função do Diâmetro Nominal (DN) da rede

\begin{tabular}{|c|c|c|c|c|c|}
\hline & \multirow{2}{*}{\multicolumn{2}{|c|}{ Custos envolvidos }} & \multicolumn{3}{|c|}{ DN } \\
\hline & & & 50 & 75 & 100 \\
\hline \multirow{5}{*}{ 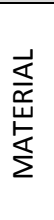 } & \multicolumn{2}{|c|}{ Tubulação PVC (m) } & 55,62 & 104,58 & 187,86 \\
\hline & \multicolumn{2}{|c|}{ Anéis de borracha (un.) } & 1,36 & 4,48 & 3,50 \\
\hline & \multicolumn{2}{|c|}{ Pasta Lubrificante (g) } & 0,32 & 0,52 & 0,63 \\
\hline & \multicolumn{2}{|c|}{ Sub-Total (R\$ por $6 \mathrm{~m})$} & 57,30 & 109,58 & 191,99 \\
\hline & \multicolumn{2}{|c|}{ Relação entre o custo do material e o custo total (\%) } & $31,9 \%$ & $45,9 \%$ & $58,5 \%$ \\
\hline & \multicolumn{2}{|c|}{ Locação da rede de distribuição de água (m) } & 4,26 & 4,26 & 4,26 \\
\hline & \multicolumn{2}{|c|}{ Volume Escavado - Ve $\left(\mathrm{m}^{3}\right)$} & 20,25 & 20,72 & 21,20 \\
\hline & \multicolumn{2}{|c|}{ Regularização e compactação do fundo de vala $\left(\mathrm{m}^{2}\right)$} & 19,15 & 19,15 & 19,15 \\
\hline 它 & \multicolumn{2}{|c|}{ Assentamento da Tubulação $(\mathrm{m})$} & 10,32 & 13,80 & 17,22 \\
\hline 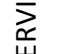 & \multicolumn{2}{|c|}{ Volume de aterro com apiloamento manual - VACM $\left(\mathrm{m}^{3}\right)$} & 54,16 & 57,21 & 60,25 \\
\hline & \multicolumn{2}{|c|}{ Volume de aterro com compactação mecânica-VACMec $\left(\mathrm{m}^{3}\right)$} & 14,11 & 14,11 & 14,11 \\
\hline & \multicolumn{2}{|c|}{ Sub-Total (R\$ por $6 \mathrm{~m})$} & 122,25 & 129,25 & 136,19 \\
\hline & \multicolumn{2}{|c|}{ Relação entre o custo dos serviços e o custo total (\%) } & $68,1 \%$ & $54,1 \%$ & $41,5 \%$ \\
\hline & \multirow{2}{*}{ Custo Total } & Por $6 \mathrm{~m}(\mathrm{R} \$)$ & 179,55 & 238,83 & 328,10 \\
\hline & & Linear $(R \$ / m)$ & 29,93 & 39,81 & 54,68 \\
\hline
\end{tabular}

\subsubsection{Comparação entre os custos de implantação de redes simples e redes duplas}

A partir das vazões máximas para cada diâmetro, determinadas em função das velocidades máximas de escoamento determinadas por SABESP (1999), determinou-se os custos lineares para implantação das redes de distribuição de água simples (assentada no terço do leito carroçável) e dupla (assentadas nos dois passeios), conforme apresentado na Tabela .

Tabela 10: Distribuição dos custos para implantação de redes de distribuição de água simples e duplas em função

\begin{tabular}{|c|c|c|c|c|c|}
\hline \multirow[b]{2}{*}{$\begin{array}{l}\text { Diâmetro } \\
\text { Nominal da } \\
\text { Rede } \\
\text { (DN) }\end{array}$} & \multirow[b]{2}{*}{$\begin{array}{c}\text { Vazão } \\
\text { máxima } \\
\text { admissível } \\
(\mathrm{L} / \mathrm{s})^{*}\end{array}$} & \multicolumn{2}{|l|}{ Rede Simples } & \multicolumn{2}{|c|}{ Rede Dupla } \\
\hline & & $\begin{array}{l}\text { Intervalo de Vazão para } \\
\text { atendimento do trecho } \\
\text { (L/s) }\end{array}$ & $\begin{array}{l}\text { Custo } \\
\text { (R\$/m) }\end{array}$ & $\begin{array}{l}\text { Intervalo de } \\
\text { Vazão para } \\
\text { atendimento do } \\
\text { trecho } \\
\text { (L/s) }\end{array}$ & $\begin{array}{l}\text { Custo } \\
\text { (R\$/m) }\end{array}$ \\
\hline 50 & 1,43 & $0,00 \nmid 1,43$ & 29,93 & $0,00 \dashv 2,86$ & 57,94 \\
\hline 75 & 3,33 & $1,43-3,33$ & 39,81 & $2,86 \dashv 6,66$ & 77,72 \\
\hline 100 & 7,69 & $3,33-7,69$ & 54,68 & $6,66 \dashv 15,38$ & 107,49 \\
\hline
\end{tabular}

Nota: * adaptado de SABESP (1999)

A Figura 2 contém os intervalos de custos para implantação de redes de distribuição de água simples (assentada no terço do leito carroçável) e dupla (assentada nos passeios) em função da vazão de projeto. 


\section{Revista Nacional de}

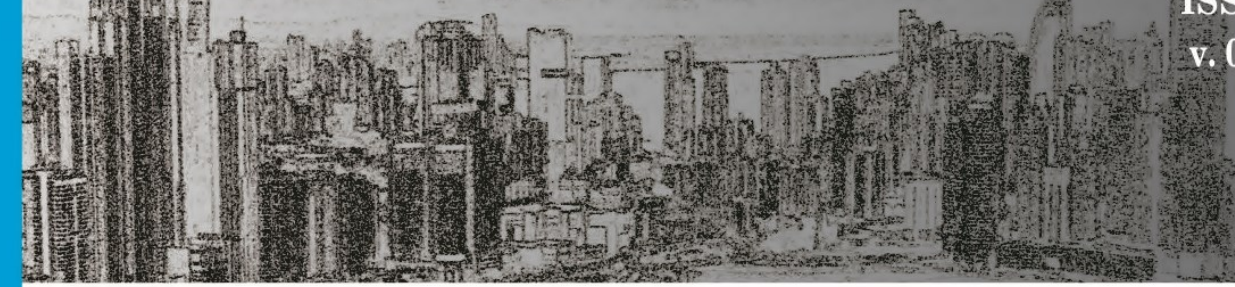

Tabela 11: Quantitativos referentes às ligações prediais executadas em redes de distribuição de água simples e duplas

\begin{tabular}{|c|c|c|c|c|c|c|c|}
\hline \multirow{2}{*}{\multicolumn{2}{|c|}{ Item }} & \multicolumn{3}{|c|}{ Ligação em Rede Simples } & \multicolumn{3}{|c|}{ Ligação em Rede Dupla } \\
\hline & & DN 50 & DN 75 & DN 100 & DN 50 & DN 75 & DN 100 \\
\hline \multirow{7}{*}{ 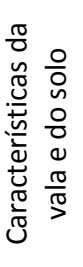 } & Largura do passeio - Lp (m) & \multicolumn{3}{|c|}{3,0} & \multicolumn{3}{|c|}{3,0} \\
\hline & Largura do Leito - LL (m) & \multicolumn{3}{|c|}{11,0} & \multicolumn{3}{|c|}{11,0} \\
\hline & Profundidade média da vala para ligação $(\mathrm{m})$ & \multicolumn{3}{|c|}{0,65} & \multicolumn{3}{|c|}{0,35} \\
\hline & Largura da vala para ligação $(\mathrm{m})$ & \multicolumn{3}{|c|}{0,30} & \multicolumn{3}{|c|}{0,30} \\
\hline & Comprimento da vala $(\mathrm{m})$ & \multicolumn{3}{|c|}{8,5} & \multicolumn{3}{|c|}{2,0} \\
\hline & Tipo de solo & \multicolumn{3}{|c|}{ 1a Categoria } & \multicolumn{3}{|c|}{ 1a Categoria } \\
\hline & Empolamento (\%) & \multicolumn{3}{|c|}{$25 \%$} & \multicolumn{3}{|c|}{$25 \%$} \\
\hline \multirow{5}{*}{$\frac{0}{\stackrel{0}{0}}$} & Locação da ligação predial (m) & \multicolumn{3}{|c|}{3,0} & \multicolumn{3}{|c|}{3,0} \\
\hline & Escavação mecânica - Ve $\left(\mathrm{m}^{3}\right)$ & \multicolumn{3}{|c|}{0,89} & \multicolumn{3}{|c|}{0,21} \\
\hline & Regularização e compactação do fundo de vala $\left(\mathrm{m}^{2}\right)$ & \multicolumn{3}{|c|}{2,55} & \multicolumn{3}{|c|}{0,60} \\
\hline & Execução da ligação da rede ao ramal predial (und.) & 1 & 1 & 1 & 1 & 1 & 1 \\
\hline & Reaterro com compactação mecânica-VACMec $\left(\mathrm{m}^{3}\right)$ & \multicolumn{3}{|c|}{2,06} & \multicolumn{3}{|c|}{0,26} \\
\hline \multirow{3}{*}{ 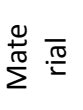 } & Colar de Tomada (uni) & 1 & 1 & 1 & 1 & 1 & 1 \\
\hline & Tubo PEAD 2mm (m) & \multicolumn{3}{|c|}{10,50} & \multicolumn{3}{|c|}{4,00} \\
\hline & Kit cavalete com registro $3 / 4 "$ & \multicolumn{3}{|c|}{1} & \multicolumn{3}{|c|}{1} \\
\hline
\end{tabular}

A partir da determinação dos quantitativos envolvidos na implantação das ligações prediais nas redes simples e duplas de distribuição de água, aplicou-se os custos estabelecidos pelo SINAPI (2015.a) e SINAPI (2015.b), conforme apresentados nas Tabelas 11 e 12, de maneira a determinar os custos de serviços e materiais, respectivamente.

$\mathrm{Na}$ Tabela apresentam-se os custos determinados para implantação das ligações de água em redes simples e duplas de abastecimento de água em função do diâmetro nominal da rede.

Tabela 12: Custos referentes às ligações prediais executadas em redes de distribuição de água simples e duplas

\begin{tabular}{|c|c|c|c|c|c|c|c|}
\hline & \multirow{3}{*}{ Item } & \multicolumn{6}{|c|}{ Custos (R\$) } \\
\hline & & \multicolumn{3}{|c|}{ Ligação em Rede Simples } & \multicolumn{3}{|c|}{ Ligação em Rede Dupla } \\
\hline & & DN 50 & DN 75 & DN 100 & DN 50 & DN 75 & DN 100 \\
\hline \multirow{7}{*}{ 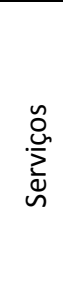 } & Locação da ligação predial (m) & & 2,13 & & & 2,13 & \\
\hline & Escavação mecânica - Ve $\left(\mathrm{m}^{3}\right)$ & & 4,72 & & & 1,11 & \\
\hline & Regularização e compactação do fundo de vala $\left(\mathrm{m}^{2}\right)$ & & 13,57 & & & 3,19 & \\
\hline & Execução da ligação da rede ao ramal predial (und.) & 36,60 & 41,18 & 50,00 & 36,60 & 41,18 & 50,00 \\
\hline & Reaterro com compactação mecânica-VACMec $\left(\mathrm{m}^{3}\right)$ & & 16,15 & & & 2,04 & \\
\hline & Sub-Total (R\$) & 73,17 & 77,75 & 86,57 & 45,07 & 49,65 & 58,47 \\
\hline & Relação entre Custo de Serviço e Custo Total (\%) & $45,4 \%$ & $46,1 \%$ & $48,3 \%$ & $33,4 \%$ & $35,3 \%$ & $38,7 \%$ \\
\hline \multirow{5}{*}{ 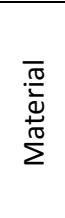 } & Colar de Tomada (uni) & 8,99 & 11,85 & 13,49 & 8,99 & 11,85 & 13,49 \\
\hline & Tubo PEAD 2mm (m) & & 46,83 & & & 17,72 & \\
\hline & Kit cavalete com registro $3 / 4$ & & 32,18 & & & 32,18 & \\
\hline & Sub-Total (R\$) & 88,00 & 90,86 & 92,50 & 88,00 & 90,86 & 92,50 \\
\hline & Relação entre Custo de Material e Custo Total (\%) & $54,6 \%$ & $53,9 \%$ & $51,7 \%$ & $66,6 \%$ & $64,7 \%$ & $61,3 \%$ \\
\hline \multicolumn{2}{|c|}{ Total Geral (R\$) } & 161,17 & 168,61 & 179,07 & 133,07 & 140,51 & 150,97 \\
\hline
\end{tabular}

Analisando os dados da Tabela é possível observar que os custos envolvidos para implantação das ligações prediais em redes duplas são inferiores àqueles obtidos para redes simples. 


\section{Revista Nacional de}

Gerenciamento de Cidades

- Para as condições estabelecidas neste trabalho, as redes duplas, com as respectivas ligações prediais, não se mostravam economicamente viáveis quando comparados aos custos globais desta com os da rede simples e suas respectivas ligações prediais.

\section{REFERÊNCIAS BIBLIOGRÁFICAS}

ABNT - ASSOCIAÇÃO BRASILEIRA DE NORMAS TÉCNICAS (1992). NBR 12.268: Projeto e execução de valas para assentamento de tubulação de água, esgoto ou drenagem urbana. Rio de Janeiro, 1992. 17p.

ABNT - ASSOCIAÇÃO BRASILEIRA DE NORMAS TÉCNICAS (1994). NBR 12.218: Projeto de rede de distribuição de água para abastecimento público - Procedimento. Rio de Janeiro, 1994. 4p.

AMANCO (2014). Catálogo Técnico: Infraestrutura. 72p. Disponível em: < http://www.amanco.com.br/web/image/texto/file/Catalogo_Infra_2014_web.pdf>

FORTILIT (s.d.). Catálogo Técnico: Infra-Estrutura.

MATTOS, Aldo Dórea. BLOG PINI. Material de 1a, 2a e $3^{\underline{a}}$ categoria. 8/julho/2014. Disponível em: http://blogs.pini.com.br/posts/Engenharia-custos/material-de-1-2-e-3-categorias-318190-1.aspx

SÃO CARLOS. LEI № 13.691 de 25 de Novembro de 2005. Institui o Plano Diretor do Município de São Carlos e dá outras providências. São Carlos, 2005a. 69p+Anexos

SABESP - COMPANHIA DE SANEAMENTO BÁSICO DO ESTADO DE SÃO PAULO. Especificação Técnica, Regulamentação e Critérios de Medição. $2^{a}$ Edição. São Paulo. SABESP, 1996. 1145P.

SABESP - COMPANHIA DE SANEAMENTO BÁSICO DO ESTADO DE SÃO PAULO. NTS 024: Rede de distribuição de água. Procedimentos. São Paulo, 1999. 3p

SÃO PAULO. DEPARTAMENTO DE ESTRADAS DE RODAGEM - DER. Especificação Técnica: Escavação e Carga de Material. 2006. 11p. Disponível em: ftp://ftp.sp.gov.br/ftpder/normas/ET-DE-Q00-002_A.pdf

SINAPI - Sistema Nacional de Pesquisa de Custos e Índices da Construção Civil (2015.a). Índice da Construção Civil/Serviços/Maio de 2015. Disponível em http://www.caixa.gov.br/Downloads/sinapi-a-partir-jul-2014sp/SINAPI_Custo_Ref_Composicoes_SP_052015_NaoDesonerado.pdf>.

SINAPI - Sistema Nacional de Pesquisa de Custos e Índices da Construção Civil (2015.b). Índice da Construção Civil/Insumos/Maio de 2015. Disponível em http://www.caixa.gov.br/Downloads/sinapi-a-partir-jul-2014sp/SINAPI_Preco_Ref_Insumos_SP_052015_Desonerado.PDF. 